$10 \mid 2006$

Varia

\title{
Auxerre (Yonne), la cathédrale Saint-Étienne
}

Christian Sapin, Sylvain Aumard, Stéphane Büttner et Heike Hansen

\section{(2) OpenEdition}

\section{Journals}

Édition électronique

URL : https://journals.openedition.org/cem/478

DOI : $10.4000 /$ cem.478

ISSN : 1954-3093

Éditeur

Centre d'études médiévales Saint-Germain d'Auxerre

Édition imprimée

Date de publication : 15 août 2006

ISSN : 1623-5770

Référence électronique

Christian Sapin, Sylvain Aumard, Stéphane Büttner et Heike Hansen, « Auxerre (Yonne), la cathédrale Saint-Étienne », Bulletin du centre d'études médiévales d'Auxerre / BUCEMA [En ligne], 10 | 2006, mis en ligne le 15 novembre 2006, consulté le 22 septembre 2022. URL : http://journals.openedition.org/cem/ 478 ; DOI : https://doi.org/10.4000/cem.478

Ce document a été généré automatiquement le 22 septembre 2022.

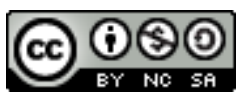

Creative Commons - Attribution - Pas d'Utilisation Commerciale - Partage dans les Mêmes Conditions 4.0 International - CC BY-NC-SA 4.0

https://creativecommons.org/licenses/by-nc-sa/4.0/ 


\title{
Auxerre (Yonne), la cathédrale Saint-Étienne
}

\author{
Christian Sapin, Sylvain Aumard, Stéphane Büttner et Heike Hansen
}

1 Dans un programme continu et croisé entre chantiers archéologiques, étude de bâti et suivi de la restauration de la cathédrale Saint-Étienne d'Auxerre, le Centre d'études médiévales, en concertation avec le Conseil scientifique de la cathédrale, poursuit ses recherches. Nous livrons comme l'an passé dans ce bulletin, les premiers résultats, en annonçant dès à présent le colloque international envisagé les 27-28 septembre 2007 à Auxerre, où sera présenté et discuté l'ensemble des travaux, des observations et des relevés des campagnes 2002-2006. Une publication complète de ces recherches est également prévue à cette occasion ainsi qu'une exposition.

Programme de recherche archéologique : la crypte

2 L'étude archéologique de la construction de la crypte $\mathrm{du} \mathrm{XI}^{\mathrm{e}}$ siècle s'est poursuivie en 2005 par le relevé et l'analyse des données bâties du mur sud de la grande salle et du déambulatoire ou couloir sud. Après avoir recueilli le maximum d'informations dans les zones occidentales pour comprendre les transformations successives des accès et avant d'engager une fouille au sol dans des secteurs appropriés de la crypte (relation avec le déambulatoire supérieur, côté sud, ou chapelle d'axe) il est apparu nécessaire de poursuivre la reconnaissance des particularités de la construction en 2005 et en 2006.

Le travail d'analyse et de dessin s'est poursuivi avec l'équipe du CEM et des stagiaires à la fois pour les maçonneries et pour les enduits et peintures.

Il est ressorti de la campagne 2005 de nouvelles remarques sur les niches et les ouvertures plusieurs fois transformées entre le couloir de déambulation et la salle avec, très rapidement, un système obturant certaines baies d'éclairage et la création d'une chapelle probablement au cours du $\mathrm{XII}^{\mathrm{e}}$ siècle dans le même couloir. Son cloisonnement est reconnaissable aujourd'hui avec la limite conservée dans le repeint d'un doubleau et d'autres indices. Les travaux de reconstruction du chœur gothique transformeront à nouveau, espace et accès avec l'aménagement d'une chapelle cette fois au sud au sud de la crypte. 


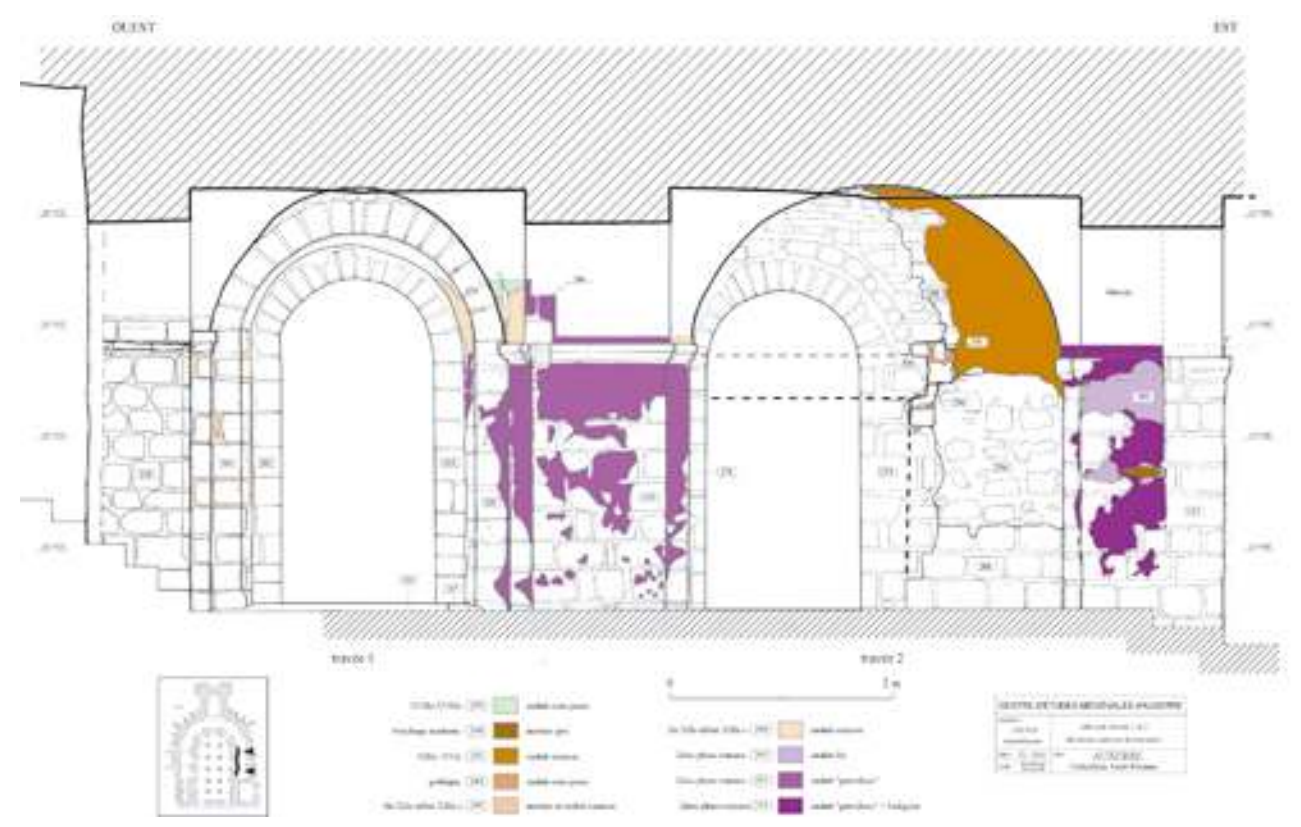

Déambulatoire sud, mur nord, $7^{\text {ère }}$ et $2^{\text {ème }}$ travées, élévation W/E (dessin, 2005, CEM)

Étude documentaire sur les portails (Sylvain Aumard)

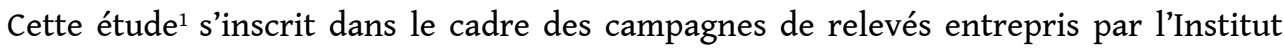
d'architecture de l'Université de Stuttgart sur les portails de la cathédrale SaintÉtienne d'Auxerre. Elle nous a été demandée dans le but de regrouper tous les indices documentaires permettant de mieux appréhender l'histoire de la construction et de l'entretien de ces ouvrages au cours du temps.

6 Pour mener à bien une telle enquête, nous avons suivi trois directions de recherche : les archives médiévales et de l'Ancien Régime, les archives des restaurations des $\mathrm{XIX}^{\mathrm{e}}$ et $\mathrm{XX}^{\mathrm{e}}$ siècles et les photographies anciennes.

7 Les travaux de recherches entrepris au cours de ces dernières décennies sur cet édifice majeur de l'art gothique avaient déjà tenté de rassembler nombre d'indices sur la progression du chantier médiéval, en particulier Harry B. Titus dans le cadre de sa thèse ${ }^{2}$. Cette démarche avait également été entreprise de façon plus ciblée sur les portails occidentaux par Ursula Quednau ${ }^{3}$. Au moment d'entreprendre notre étude, la documentation médiévale et moderne avait donc été largement repérée pour l'essentiel, d'autant que d'autres études plus anciennes cette fois, avaient aussi largement progressé dans cette direction, notamment sous les plumes de Charles Porée et de l'abbé Lebeuf ${ }^{4}$. Ainsi, en ce qui concerne la documentation médiévale et moderne, il était difficile d'espérer découvrir d'autres pistes inexplorées jusqu'alors. Nous avons pu aussi bénéficier de la réédition récente de l'Histoire de la prise d'Auxerre par les Huguenots ${ }^{5}$, source peu exploitée jusqu'ici semble t-il. Grâce à la présence, au Centre d'études médiévales, du groupe œuvrant à la traduction et à l'édition des Gesta Pontificum ${ }^{6}$, nous avons eu la primeur de bénéficier de ce travail. Ainsi, a-t-il été possible de recouper cette source avec les indications de Lebeuf et de mieux apprécier la portée de chacun de ces documents.

8 L'inventaire de la série $G$, conservée aux Archives départementales de l'Yonne, a été consulté intégralement pour ce qui concerne les archives du chapitre cathédral ${ }^{7}$. Si aucune découverte substantielle n'a été faite, on peut émettre l'hypothèse qu'il y ait peu de chance de repérer autre chose dans cette série au cours de recherches 
ultérieures. Cet inventaire a en effet été rédigé par Maximilien Quantin. Cet archiviste a porté une attention significative au classement des comptabilités des chanoines de la cathédrale de Sens inventoriées dans la même série, en les traitant à l'égal des autres documents et en publiant de nombreux extraits dans son inventaire. Quantin a donc connaissance de ce genre de source et ainsi de leur portée historique intrinsèque. Il y a donc fort à parier que s'il avait rencontré pareille documentation pour Auxerre, il l'aurait signalée.

On rappelle seulement ici les principaux faits relatés par la documentation écrite en rapport avec la construction des portails :

- sur la façade ou le portail central, mention des travaux de sculpture en 1397 et de la fabrication des ventaux en $1403^{8}$;

- en avant-corps du portail sud, mention de la chapelle Notre-Dame-des-Vertus dès $1361^{9}$ où se déroule un miracle relaté en 1398. Cette chapelle sera déplacée au sud de la tour méridionale au milieu du xvie siècle ;

- sur le transept nord, mention de l'achat des portes en $14222^{10}$.

10 La documentation des Monuments historiques consultée à Auxerre, aux Archives départementales (série T), ou à Paris, à la Médiathèque du patrimoine, fait apparaître que beaucoup de travaux sont manifestement restés à l'état de projet : dans ces cas, on ne dispose généralement que de devis ou d'états des lieux et aucun document ne permet de s'assurer que les ouvrages proposés ont été réalisés. Les fonds d'archives non consultés ou inaccessibles, tels que ceux des Archives municipales et des architectes départementaux, permettraient assurément de mieux préciser ces aspects. Par ailleurs, celles-ci permettraient aussi de mieux appréhender certaines de ces réparations mal documentées que les observations de terrain montrent comme probablement réalisées. Le principal intérêt de ces projets avortés est l'état des lieux qu'ils présentent à une date donnée. On peut ainsi proposer une datation avec un terminus ante quem pour certaines dégradations actuellement perceptibles et constatées par les restaurateurs du XIX ${ }^{e}$ siècle : par exemple, les socles des ébrasements du portail du transept sud étaient déjà à réparer en 1843 et ne l'ont toujours pas été depuis.

11 Les travaux dont l'exécution est explicitement attestée par certains documents (mémoires, métrés, règlements de compte, autorisations de travaux...) demeurent peu nombreux. Pas toujours localisables, leur nature exacte n'est pas systématiquement identifiée. Les mieux documentés se situent au transept nord en 1840 et 1943-1946, au transept sud en 1908 et sur les portails occidentaux en 1945. Ceux des années 1945 et 1946 concernent le dépoussiérage et le rescellement ponctuel de la sculpture: le mémoire de maçonnerie de l'entreprise Chatignoux, daté du 20 novembre 1942, indique notamment qu'une vingtaine "de petits morceaux de pierre sculptés des dais jusqu'à $0,15[\mathrm{~m}]$ » ont été refixés à l'aide de ciment et de gougeons en fer, après « recherche préalable parmi les fragments (...) du musée " ${ }^{11}$. Les autres interventions ne sont pas autant localisables, aussi l'étude géologique des pierres de construction et leur cartographie sur les relevés de l'Université de Stuttgart ${ }^{12}$ permettent cependant de s'en forger une idée plus précise :

- ceux de 1840 se lisent très bien sur les parements nord et ouest à l'intérieur du transept nord ;

- ceux de 1908 semblent en réalité avoir été largement réduits par rapport au projet initial, car ils pourraient se limiter à la reprise en sous-œuvre du trumeau du portail (calcaire à oolithes et entroques). 
D'autres travaux attestés sur la façade occidentale, dont la localisation et la nature ne sont pas précisément indiqués par les textes, peuvent désormais être mieux cernés, tels ceux de 1831 au portail nord et ceux d'avant 1837 au portail central. Si, d'après les textes, on savait qu'il s'agissait de réparations au socle, on peut désormais préciser qu'il s'agit en réalité de la première assise des élévations formant le tout premier ressaut (calcaire à oolithes et entroques là aussi).

13 En revanche, les travaux "non achevés » en 1830 ne se laissent guère percevoir. On peut toutefois se demander si finalement la réparation du trumeau du transept sud ne peut pas remonter à cette époque car le calcaire employé ici est identique à celui utilisé en 1831 pour le socle du portail nord de la façade.

Les archives de la Médiathèque du patrimoine et de la DRAC montrent également que les portails occidentaux ont fait l'objet d'un projet de nettoyage par Bernard Collette. Une étude du Laboratoire de recherches des Monuments historiques a été commanditée dans ce cadre pour déterminer l'origine de l'humidité. Dans son rapport du 12 mai 1980, le laboratoire préconise la pose d'un drain qui sera réalisé en 1982 après un sondage pratiqué au pied de l'ébrasement nord du portail central.

列 la cathédrale d'Auxerre, dont 210 sur les portails (tympan, voussures, ventaux et face interne). On remarque aisément que le portail du transept nord est sous représenté par rapport aux autres, probablement en raison de sa sculpture tardive. Deux principaux aspects de l'histoire des portails peuvent être appréhendés :

- la trace de l'oratoire Notre-Dame-des-Vertus sur la façade occidentale, sous la forme d'une ligne blanche et oblique, visible sur le parement du contrefort ouest, témoignant de la pente du toit disparu (photographies entre 1851 et 1909) ;

- les tringles métalliques servant à suspendre des draps mortuaires aux portails sud et central de la façade occidentale ; elles sont attestées dès les années 1880 , puis dans le courant du xxe siècle ; elles ont notamment été réparées au cours des travaux de nettoyage réalisés en 1942 par Jean Trouvelot ${ }^{13}$; leur présence sur les clichés pris par Ursula Quednau au cours de sa thèse dans les années soixante dix, indique qu'elles ont été déposées au cours des dernières décennies ${ }^{14}$.

Le suivi de travaux de restauration et les analyses. Premiers résultats.Étude géologique des pierres de construction des chantiers gothiques (Stéphane Büttner)

A priori, l'identification géologique des pierres de construction de la cathédrale pouvait présenter un certain intérêt quant à la compréhension de la succession des phases de chantier. Quelques études antérieures avaient déjà révélé l'utilisation de faciès variés de manière très localisée dans la construction. De fait, la pierre pouvait être un marqueur chronologique. En retour, la mise en perspective de cette étude pouvait apporter des éléments de compréhension déterminants quant à l'économie de la pierre icaunaise au Moyen Âge.

L'entreprise engagée visait donc à situer clairement dans le bâti les différents faciès géologiques reconnus en s'aidant pour cela des relevés archéologiques réalisés par l'équipe de l'Institut d'Architecture de l'Université de Stuttgart ${ }^{15}$. Des cartographies lithologiques précises ont pu être ainsi réalisées à la faveur des différents échafaudages disposés pour la restauration de l'édifice. Des contrôles ont pu être également effectués à l'aide d'une nacelle élévatrice, tant sur les élévations extérieures qu'à l'intérieur de la nef.

Bulletin du centre d'études médiévales d'Auxerre | BUCEMA, 10 | 2006 
ne attention particulière a été portée sur la façade occidentale et ses portails (parements intérieur et extérieur). Dès 1985, cette zone avait déjà fait l'objet d'analyses ponctuelles réalisées par le Laboratoire de Recherche des Monuments Historiques ${ }^{16}$; cette nouvelle étude vient les compléter. De même, des investigations ont été menées sur le portail et les élévations du transept sud, tant à l'extérieur qu'à l'intérieur. Le portail et les élévations du transept nord ont fait l'objet de la même attention. Enfin, une attention particulière a été portée sur les piliers des arcatures de la nef où l'emploi de la pierre parisienne s'avérait très important.

\section{transept avec la construction des deux piles occidentales de la croisée. Les deux piles de} la dernière travée de la nef semblent également être amorcées. Une nouvelle fois, ce sont les faciès du Jurassique qui sont utilisés (Bajocien-Bathonien). Le portail du transept sud semble également être édifié lors de cette période. On remarquera, à l'instar des portails de la façade occidentale, que le faciès type "pierre de Tonnerre » a été sans doute préféré pour les éléments sculptés puisqu'il constitue l'essentiel du tympan et des voussures de ce portail. avec la construction des piliers. Il est alors intéressant de voir que ceux-ci sont intégralement constitués de calcaire lutétiens parisiens jusqu'à la base du triforium. Les arcs des bas-côtés, ainsi que toute la construction qui se situe au-delà, sont composés de « pierres de Tonnerre ».

25 Cette approche nous permet donc d'aborder deux points. L'un concerne la nature et l'origine des approvisionnements, l'autre l'adéquation éventuelle entre nature des matériaux et fonctions.

Ainsi, d'une manière générale, on constate que lors de la première moitié du XIII ${ }^{\mathrm{e}}$ siècle, ce sont essentiellement les faciès bathoniens-bajociens qui ont été sollicités. Provenant

Bulletin du centre d'études médiévales d'Auxerre | BUCEMA, 10 | 2006 
d'une trentaine de kilomètres au sud d'Auxerre, ces pierres constituent en effet l'essentiel du moyen appareil. Puis, à partir de la seconde moitié du XIII ${ }^{\mathrm{e}}$ siècle on constate un apport conséquent de pierres parisiennes, tout d'abord en l'espèce du Liais, mais aussi du Lutétien plus classique à Milioles et Cérithes. Si, aux vues de son origine lointaine, l'emploi de cette dernière pierre ne se justifie pas du fait des similitudes physico-mécaniques avec les faciès de l'Auxerrois traditionnellement utilisés dans la construction locale, l'emploi du Liais semble répondre, quant à lui, à un choix délibéré d'une pierre dure et résistante dont l'emploi semble se généraliser dans les fondations des grands monuments du bassin parisien de cette période ${ }^{17}$.

Aux XIII ${ }^{\mathrm{e}}$ siècle, l'emploi de la "pierre de Tonnerre" (Kimméridgien) semble se cantonner aux éléments sculptés. En effet, d'aspect crayeux et tendre, cette pierre se prête extrêmement bien à ce type de travail.

On observe un nouvel apport de pierre de la région parisienne au milieu du XIve siècle (piliers de la nef). Cet emploi semble bel et bien correspondre à la mention, datée de 1341, indiquant que Jean de Valrenfroy, qui a dirigé à la fois le chantier de Sens et celui d'Auxerre, fit venir des pierres d'Ivry pour le compte de ces deux chantiers ${ }^{18}$.

À partir de la seconde moitié du xIv siècle, l'emploi de la « pierre de Tonnerre » pour le moyen appareil semble se généraliser dans les mêmes fonctions qu'occupait la pierre bajociennes-bathoniennes lors du siècle précédent. Plus proche, il est possible que les exploitations des bords de l'Yonne (région de Bailly) connaissent un essor important lors de cette période, justifiant leur sollicitation au détriment des carrières plus éloignées de la région.

En intégrant ces observations à une réflexion plus globale sur l'économie de la pierre icaunaise à l'époque gothique, il apparaît que ces importations de pierres parisiennes, techniquement incongrues, peuvent s'expliquer par une pénurie de pierres autochtones. Non pas que l'exploitation locale cesse, mais il est très plausible que le rythme soutenu des chantiers gothiques de la région, et le volume important de pierres alors nécessaires, font que la production locale ne suffit plus. C'est donc à la fin du XIV que les carrières de la région vont connaître un nouvel essor important, ce qui coïncide peut-être avec leur exploitation en souterrain. Quoiqu'il en soit cette production se pérennise jusqu'au début du $\mathrm{Xx}^{\mathrm{e}}$ siècle.

Les datations des charpentes des bas-côtés nord et sud, de la tour sud et de la chapelle de l'oratoire (suite) (Sylvain Aumard)

Hormis celle de l'oratoire, nous ne reviendrons pas sur la description sommaire de ces charpentes déjà évoquées dans le Bulletin de l'an passé ${ }^{19}$. Nous avions annoncé qu'une étude dendrochronologique devait livrer ses résultats dans le courrant de l'année $2005^{20}$. Nous en livrons ici les principaux acquis.

La charpente des bas-côtés nord présente deux états succédant à un premier ayant disparu. Un système à portique, situé au-dessus des chapelles et paraissant en rapport avec leur construction, a ensuite été complété, au-dessus des bas-côtés, par un système à fermes et pannes à profil en « V ». Lors de la dépose de l'ouvrage en juin 2004, quatorze prélèvements sous la forme de tronçons ont été réalisés par les charpentiers ${ }^{21}$ à notre demande. Au cours de l'étude dendrochronologique, sept prélèvements sous la forme de carottage ont complété le corpus. La chronologie relative de ces charpentes a été confirmée et la présence du dernier cerne de croissance sur la plupart des prélèvements permet désormais d'avoir une idée très précise de leur mise en œuvre. 
Les bois du dernier état ont été abattus au cours de l'automne-hiver 1820-1821. Parmi ceux du second état, un seul a pu être daté de l'automne-hiver 1357-1358. Cette dernière datation montre en réalité que cette pièce de bois (faîtage) a été utilisée en remploi, car on sait que la chapelle sous-jacente a été élevée entre 1379 et $1412^{22}$. Il s'agit donc très vraisemblablement d'une pièce ayant appartenu initialement à la charpente des bas-côtés nord dont les bois auraient ainsi été coupés un an environ après ceux du premier module du grand comble de la nef datés de 1356. L'analyse du profil dendrologique de cet élément de faittage montre, en outre, qu'il provient probablement du même bassin forestier que celui des bois de 1356.

Sur les bas-côtés sud, vingt-cinq échantillons ont été prélevés par carottage. Le module de charpente appuyé contre le transept n'a pu être pris en compte à la fois en raison de sa fragilité apparente et de la faible section de ses pièces de bois qui n'aurait pu apporter des données suffisamment exploitables. Nous nous contenterons donc d'une estimation de sa datation aux environs $d u x \mathrm{xVIII}^{\mathrm{e}}$ siècle basée sur la grande médiocrité de sa réalisation. Les ouvrages de noues situés dans les angles avec le transept et la tour sud sont les plus récents : la coupe de leur bois est intervenue au cours des automnes-hivers 1828 et 1829. Ils sont donc venus en remplacement de structures plus anciennes, vraisemblablement en rapport avec le module central, et dont la conception devait être déficiente pour permettre une évacuation efficace des eaux de pluie aux extrémités des bas-côtés. L'ouvrage central, quant à lui, n'a pu être daté car l'hétérogénéité du profil dendrologique de ses bois induit un risque d'erreur statistique particulièrement élevé pour toutes propositions. On doit se contenter d'une fourchette de datation comprise entre le milieu du XvI ${ }^{\mathrm{e}}$ siècle (achèvement de la nef et de la façade) et la fin du XviII ${ }^{e}$ siècle. Les quelques tronçons repérés sur place en vue de leur prélèvement au cours de l'opération de dépose (en 2006), pourront peut-être résoudre cette question. Les charpentes des chapelles ne présentaient pas de bois pouvant se prêter à une étude dendrochronologique; cependant, rien n'interdit de penser que leur charpente était d'une conception proche de celle rencontrée au-dessus des chapelles nord (système à portique ?).

La tour sud est un ouvrage inachevé $\mathrm{du} \mathrm{XvI}{ }^{\mathrm{e}}$ siècle dont le toit à versant unique repose sur une charpente à fermes et pannes paraissant remonter aux XVII ${ }^{\mathrm{e}}$-XVIII ${ }^{\mathrm{e}}$ siècles. Cette dernière a remplacé un premier ouvrage provisoire destiné à protéger les maçonneries et dont les seules traces ont été relevées dans la corniche sud sous la forme d'encoches. Les études dendrochronologiques menées à partir de seize carottages apportent une précision sensible à la datation avancée à partir du diagnostic archéologique : les bois ont été abattus au cours des automnes-hivers 1636 et 1637.

La chapelle de l'oratoire a été rajoutée au XVIII ${ }^{\mathrm{e}}$ siècle contre le mur est de la chapelle Notre-Dame-des-Vertus élevée au $\mathrm{XvI}^{\mathrm{e}}$ siècle au pied de la tour sud. Sa charpente se compose de deux demi-fermes supportant des pannes, le tout rigidifié par une structure de contreventement disposée à l'ouest. Une petite enrayure complète le dispositif dans l'angle nord-est. La présence de marquages dont la numérotation est discordante avec celle des fermes, nous avait incité à déduire qu'il s'agissait peut-être là de remplois en provenance de la chapelle de l'oratoire Notre-Dame-des-Vertus dont les textes situent la reconstruction un peu avant $1564^{23}$. Seule leur étude dendrochronologique devait permettre de trancher.

En tout, quinze carottages ont été prélevés. La moitié d'entre eux présente le dernier cerne de croissance permettant ainsi de dater l'abattage à la saison près. Á l'analyse 
dendrochronologique, trois phases d'abattage se distinguent: automne-hiver 1740-1741, automne-hiver 1741-1742 et automne 1742-1743. Contrairement aux espérances, les bois de remploi proviennent des arbres abattus pendant l'automnehiver 1740-1741. À titre d'hypothèse, on pourrait se demander s'ils ne correspondent pas à l'élaboration d'un projet avorté sur place ou sur un autre chantier dont les matériaux auraient été revendus. La dernière coupe a probablement été mise en œuvre au cours de l'année 1743, voire au cours de 1744 comme semble l'indiquer le graffiti découvert sur le mur ouest « Roux 1744 ». Jusque-là, rien ne permettait de rattacher cette inscription à des interprétations objectives (visiteur de passage ? artisan ?).

La couverture des bas-côtés sud (Sylvain Aumard)

37 Les résultats positifs obtenus au cours des expériences menées sur les autres parties de l'édifice (grand comble de la nef, tour sud et bas-côtés nord), nous ont encouragé à proposer un suivi de la dépose des couvertures des bas-côtés sud. Réalisée en janvier 2006 , ce dernier a permis d'isoler une cinquantaine de tuiles parmi lesquels trois types nous sont désormais familiers :

- tuiles sans crochet épaisses avec pureau brut ou glaçuré vert $(35 \mathrm{x} 19 \mathrm{~cm})$;

- tuiles à crochet à pâte beige, avec pureau glaçuré vert (33 x $19 \mathrm{~cm})$;

- tuiles à crochet à pâte rouge et bord chanfreiné, avec pureau glaçuré jaune ou marron $(33 \times 19 \mathrm{~cm})$.

Les deux premiers types sont morphologiquement très proches de ceux rencontrés sur le grand comble de la nef et datés du XIV ${ }^{\mathrm{e}}$ siècle par les études archéomagnétiques de l'Université de Rennes en 2004. Le troisième, s'il a déjà été rencontré au cours de précédentes études, n'a pu jusque-là être daté ; on peut estimer, cependant, une période de production proche du XIV siècle en raison du traitement glaçuré de son pureau qui devait vraisemblablement fonctionner de paire avec les pureaux glaçurés verts.

L'intérêt de conserver et d'étudier ces éléments tient au fait qu'on ignore s'ils ont été réellement produits en même temps que ceux du grand comble ou à un autre moment du chantier. Leurs datations en laboratoire, et notamment l'étude de leurs propriétés archéomagnétiques, seraient susceptibles un jour de faire avancer cette problématique. C'est une question d'autant plus intéressante que l'analyse de la charpente n'a pas permis de déterminer à quel moment les bas-côtés sud ont été couverts (cf. supra).

Un quatrième type a été mis en évidence. Il s'agit de tuiles à crochet avec un pureau brut dont la morphologie est très proche de celle des modèles à pureau glaçuré. L'étude de ces éléments permettra de savoir si la couverture décorée de la cathédrale était composée exclusivement de tuiles glaçurées ou si elles associaient aussi des tuiles brutes, qualifiées de «blanches» dans les textes.

Études de pièces de bois scellées dans des maçonneries : boulins d'échafaudage et linteau (Sylvain Aumard, Heike Hansen)

41 Le travail de collaboration avec Heike Hansen (université de Stuttgart) s'est poursuivi cette année autour de l'analyse chronologique du chantier de la cathédrale. Plusieurs pièces de bois conservées dans des élévations ont retenu notre attention car leur 
datation était susceptible d'approvisionner la réflexion en cours. Après relevé et photographie, trois éléments ont été prélevés :

- un boulin d'échafaudage situé dans le parement ouest du contrefort sud-ouest du transept sud dont la mise en place, d'après la chronologie du bâti, serait de la fin du xIIIe siècle ou du début du xive siècle ;

- le linteau d'une porte abandonnée, dont la situation dans l'escalier ouest du transept sud semble être en relation avec un arrêt de chantier de la fin du xIIIe ou du début du xive siècle. Ce dernier marquerait la fin de l'édification du portail et précéderait celle de la partie supérieure (grande verrière). La datation de cette pièce serait soit identique à celle du boulin prélevé plus bas dans la construction, soit un peu plus tardive ;

- un autre boulin, découvert dans le mur nord de la tour sud, ne présentait pas - lui d'intérêts pour dater le bâti environnant du fait qu'il s'agit d'une pièce de charpente en remploi (jambette ?). Il était en revanche susceptible d'être en rapport avec une charpente beaucoup plus ancienne, démolie au xive siècle lors de l'édification de la partie haute de la façade et des travées occidentales de la nef : charpente de l'ancien oratoire Notre-Dame-desVertus attesté dès $1361^{24}$ ou celle protégeant les portails montés au cours de la seconde moitié du xIIIe siècle.

La mauvaise qualité $\mathrm{du}$ bois (nœuds) et le faible nombre de cernes du linteau interdisent toute datation dendrochronologique et invitent à préférer le radiocarbone ${ }^{25}$. Les deux autres éléments ont, en revanche, conservé suffisamment de cernes périphériques en bon état et les résultats sont largement à la hauteur de nos attentes. Le boulin du transept sud ayant conservé son dernier cerne de croissance, sa date d'abattage peut être située au cours de l'hiver 1310-1311. Les dendrochronologues affirment même qu'il a été mis en place dans la maçonnerie très peu de temps après sa coupe car il présente un type de champignon se développant spécifiquement sur des bois dont le taux d'humidité n'a pas été suffisamment abaissé avant d'être noyé hermétiquement dans le mortier. Cette datation confirme le montage de la partie basse du transept sud et de son portail aux environs de 1310, comme Peter Kurman l'avait déjà suggéré pour la sculpture de ce dernier. Si le second boulin d'échafaudage n'a pas conservé son dernier cerne de croissance, il possédait suffisamment d'aubier pour affirmer qu'il provient d'un arbre abattu dans le courant des années 1240-1250. Compte tenu de ce que l'on attendait, cette datation peut paraître un peu trop précoce par rapport à celle de la sculpture des portails occidentaux. En réalité, il faut également imaginer - à titre d'hypothèse - que les ouvrages ont pu être rapidement protégés par une structure charpentée, avant que ne soit amorcée la sculpture. Il faut cependant rester prudent quant à ce genre d'interprétation car cette pièce de bois peut aussi provenir d'un autre contexte dont on ignore tout (réparation d'une partie romane encore en élévation? édifice voisin, tel que Saint-Jean-Baptiste démoli également au $\mathrm{XVI}^{\mathrm{e}}$ siècle ?).

43 L'obtention de tels résultats est, d'après le dendrochronologue Didier Pousset, extrêmement rare. Les boulins d'échafaudage ne sont pas, en effet, tous nécessairement en chêne, matériaux pour lequel on dispose de solides référentiels dendrochronologiques. De plus, leur section peut être relativement faible avec peu de cernes : dans le cas du prélèvement du transept sud, on ne dispose que de 30 cernes de croissance, ce qui est très inférieure à la cinquantaine généralement requise pour obtenir une datation fiable. Si on a pu parvenir à un résultat de cette qualité, c'est bien grâce aux différents recoupements que permettent désormais les nombreux travaux 
dendrochronologiques menés en Bourgogne et notamment à Auxerre. Sur le site de la cathédrale Saint-Étienne, où la quasi-totalité des charpentes a été étudiée, plus de 260 échantillons ont été prélevés. Selon les dendrochronologues, il s'agit d'un corpus exceptionnel et sans équivalent pour un monument de ce statut, témoin historique des interactions entre la forêt et les hommes sur une période de sept siècles.

Observations sur les fenestrages du bas-côté nord (Sylvain Aumard)

La restauration du bas-côté nord a nécessité le démontage des réseaux de fenestrage du $\mathrm{XIV}^{\mathrm{e}}$ siècle des chapelles Saint-Joseph et Saint-Pélerin ( $4^{\mathrm{e}}$ et $5^{\mathrm{e}}$ travée). L'observation de ces parties, et notamment de leur mouluration, montre que les remplages ne sont pas dans leur position d'origine. Dans un premier temps, ils devaient vraisemblablement éclairer le bas-côté de la nef (ente 1324 et 1356), avant que celui-ci ne soit percé pour créer les chapelles entre les culées d'arc boutant. Ces modifications ont du avoir lieu entre 1379 et 1412 (cf. note supra). La singularité de ces éléments réside dans leur technique de mise œuvre déjà rencontrée en d'autres points du chantier du XIV siècle : leur liaisonnement a été assuré au moyen de goujons en fer noyés dans du plomb. Ce choix technique n'a pas été sans poser de problème lors du démontage car il assure une cohésion impossible à défaire. Les traces de scie repérées 5 à $10 \mathrm{~cm}$ à côté des joints au plomb indiquent bien que les constructeurs ont été obligés de scier les éléments de remplage afin de pouvoir les déplacer plus loin. Le mode de scellement employé au cours du remontage semble avoir associé pour partie la technique du métal (goujon et plomb) et pour partie celle du mortier. Le mauvais état de conservation de certains éléments empêche cependant d'être catégorique sur cette question. Cette réutilisation du fenestrage des bas-côtés explique ainsi l'apparence double de ces joints, ainsi que finalement - leur fragilité mécanique ayant poussé le restaurateur à remplacer l'intégralité de ces réseaux. Ces derniers ont été déposés dans les locaux des services techniques de la Ville, afin de poursuivre sereinement les observations nécessaires et d'étudier une solution pédagogique pour présenter une partie de ces éléments au grand public.

\section{NOTES DE FIN}

1. Étude commanditée et financée par l'Université de Stuttgart (Institut d'Architecture).

2. H. Titus, The Architectural History of Auxerre Cathedral (France), Princeton University, 1984, $445 \mathrm{p}$.

3. U. Quednau, Die Westportale der Kathedrale von Auxerre, Wiesbaden, F. Steiner Verlag, 1979, $134 \mathrm{p}$.

4. C. Porée, La cathédrale d'Auxerre, Paris, 1926 (Petites monographies des grands édifices de la France) ; J. Lebeuf (abbé), Mémoires concernant l'histoire civile et ecclésiastique d'Auxerre et de son ancien diocèse, [Paris, chez Durand, 1743], continués jusqu'à nos jours avec addition de preuves et annotations par A. Challe et M. Quantin, Auxerre, Perriquet, 1848-1855, 4 vol., LXII-546, 550, 622 et 486 p. 
5. J. Lebeuf (abbé), Histoire de la prise d'Auxerre par les huguenots et de la délivrance de la même ville, les années 1567-1568, rééd. DEGA, 2004, 294 p.

6. Nous remercions vivement Michel Sot, responsable de ce groupe de travail, de nous avoir permis d'accéder au manuscrit du second volume de l'édition en cours des Gesta. 7. M. Quantin, Inventaire-sommaire des archives départementales antérieures à 1790, Yonne, t. 2 : Archives ecclésiastiques - série G, Auxerre, 1873, VIII-456 p.

8. Respectivement : J. Lebeuf (abbé), Prise d'Auxerre... op. cit., fol. 51 [p. 57], n. 208 : Regist. Capit. ; Arch. dép. Yonne, G 1798 : conclusions capitulaires.

9. J. Lebeuf (abbé), Mémoires..., t. 1, p. 531-532 et t. 4, preuve n 340.

10. H. Titus, op. cit., preuve $n^{\circ} 39$.

11. Médiathèque du patrimoine, $81 / 89 / 10 / 2$ (carton 5), article 23.

12. S. Büttner, «Étude des pierres de construction des structures gothiques de la cathédrale Saint-Étienne d'Auxerre ", rapport inédit, Auxerre, CEM, 2005.

13. Le mémoire de maçonnerie du 20 novembre 1942 mentionne très explicitement leur réparation (Médiathèque du patrimoine, 81/89/10/2 - carton 5).

14. U. Quednau, op. cit., fig. 84, pl. 43.

15. Etude financée et commanditée par la Deutsche Forschungsgemeinschaft et la ville d'Auxerre.

16. A. Blanc, « Les monuments de l'Yonne et le commerce de la pierre au Moyen Âge ", in Bulletin de la Société des Fouilles Archéologiques et des Monuments Historiques de l'Yonne, 22 (2005), p. 9-16.

17. A. Blanc, C. Lorenz, M. Viré, « Le liais de Paris et son utilisation dans les monuments ", in Carrières et constructions en France et dans les régions limitrophes, 115e Congrès national des Sociétés savantes (Avignon), 1991, p. 247-260.

18. Arch. dép. Yonne, G 1126. C. Poree, « Les architectes et la construction de la cathédrale de Sens ", in Congrès archéologique de France (Avallon), 1908, p. 559-578.

19. Bulletin du Centre d'études médiévales d'Auxerre, Études \& travaux, CEM 9 (2004-2005), p. 27-28.

20. Étude commanditée et financée par la Ville d'Auxerre. Dendrochronologie : Didier Pousset et Christine Locatelli, Dendrochronology consulting, 8 Carr Road, Sheffield, S6 2WZ - Royaume Uni (nov. 2005).

21. Prélèvements réalisés grâce à l'aimable concours de l'entreprise Perrault (Angers) que nous remercions.

22. Il s'agit de la chapelle Saint-Pèlerin dont la chronologie du bâti indique qu'elle a été élevée après la fondation de la chapelle Saint-Joseph en 1379 (H. Titus, op. cit., preuve $\mathrm{n}^{\circ} 29$ et 41) et avant celle de la chapelle Saint-Sébastien en 1412 qui lui est contiguë à l'est (Ibid., preuve $\mathrm{n}^{\circ} 48$ ).

23. On le sait par un marché passé entre les chanoines et André Louat, le 7 mai 1564, pour réaliser la couverture (E. Drot, « Recueil de documents tirés d'anciennes minutes de notaires ", in Bulletin de la Société des sciences historiques et naturelles de l'Yonne, 53 (1889), p. 155).

24. J. Lebeuf (abbé), Mémoires... op. cit., t. 1, p. 531-532.

25. Cette analyse sera entreprise au courant de l'année 2006 et sera présentée dans le prochain Bulletin du CEM. 
INDEX

Index géographique : France/Auxerre

Mots-clés : cathédrale, charpente, crypte, fenestrage, pierre à bâtir, portail, tuile 